Results Exposure to any organic dust was associated with an increased risk of COPD in men (HR 1.32, 95\% CI: 1.12 to 1.56), and in women (HR 1.21, CI: 1.00 to 1.46). Among organic dusts, men exposed to paper dust had a HR of 1.39 (CI: 1.01 to 1.92 ). In women, paper dust (HR 1.56, CI: 1.08 to 2.26 ) and other organic dust (HR 1.48, CI: 1.12 to 1.96 ) were associated with an increased risk.

Exposure to any inorganic dust was associated with an increased risk in men (HR 1.39, CI: 1.15 to 1.67) and among these, risks for respirable crystalline silica dust (HR 1.46, CI: 1.13 to 1.90 ) and other inorganic dust and fibres (HR 1.56, CI: 1.18 to 2.05 ) were increased. Analysis of women exposed to inorganic dusts were not possible due to too few subjects. Conclusion This study confirms an increased incidence of COPD in Swedish workers exposed to organic and inorganic dusts. Health care personnel should consider not only smoking but also occupational exposure when informing COPD-patients of potential risk exposures.

\section{SILICOSIS, ESPECIALLY COMPLICATED, SHOWS SIGNIFICANT LUNG VOLUMES AND AIRFLOWS IMPAIRMENT COMPARED TO INTERSTITIAL LUNG DISEASES OF NON-OCCUPATIONAL AETIOLOGY}

S Perečinský*, Z Mačudová, L' Legáth. Department of Occupational Medicine and Clinical Toxicology, Faculty of Medicine, P. J. Šafárik University and L. Pasteur University Hospital Introduction, Košice, Slovakia

\subsection{6/oemed-2018-ICOHabstracts. 1243}

Introduction Silicosis belongs to the interstitial lung diseases, a heterogeneous group of many diseases of a different aetiology. Common symptom of all these diseases is dyspnoea. Lung function tests usually show restrictive defect with decreased diffusion capacity (DLCO). However, the heterogeneity of these diseases brings a question, if silicosis, a typically occupational disease, differs from other non-occupational interstitial diseases in the pathogenesis of symptoms and lung function impairment. The aim of study was comparison of lung functions in patients with silicosis and non-occupational interstitial lung diseases.

Methods In total, 30 patients with simple silicosis, 32 patients with complicated silicosis and 30 patients with non-occupational interstitial lung diseases underwent spirometry, bodypletysmography and DLCO examination. We compared vital capacity (VC), forced expiratory volume in one second $\left(\mathrm{FEV}_{1}\right), \mathrm{FEV} 1 / \mathrm{VC}$ ratio (Tiffeneau index), total lung capacity (TLC), residual volume (RV) and DLCO between these 3 groups of patients.

Results Vital capacity was more decreased in silicosis, both simple and complicated compared to the non-occupational interstitial lung diseases $(\mathrm{p}<0.001)$. However, TLC was the highest in complicated silicosis because of high residual volume which did not change significantly in non-occupational interstitial lung diseases $(\mathrm{p}<0.001)$. In the group of patients with silicosis, the decrease of airflows has been shown. The highest decrease of DLCO occurred in patients with non-occupational interstitial lung diseases $(63 \%)$ in comparison to simple $(p<0.001)$ and complicated $(p<0.01)$ silicosis. Only $10 \%$ of patients with non-occupational interstitial lung diseases had normal DLCO compared to $75 \%$ in simple silicosis and $45 \%$ in complicated silicosis.
Discussion Study confirmed differences in the pathogenesis of symptoms in silicosis and patients with non-occupational interstitial lung diseases. Apparently, in the case of simple silicosis, the symptoms are the result of bronchial obstruction without severe lung parenchyma destruction. In the case of non-occupational interstitial lung diseases, a predominant sing was decreased DLCO as a result of alveolocapillary membrane impairment without significant changes in lung volumes and airflows. However, complicated silicosis was characterised by significant volumes and airflows impairment and lung hyperinflation with decreasing DLCO.

\section{RESPIRATORY SYMPTOMS AND ILLNESSES AMONG BRICK KILN WORKERS IN KATHMANDU VALLEY, NEPAL}

${ }^{1}$ SK Joshi, ${ }^{2}$ S Sanjel, ${ }^{3}$ SN Khanal, ${ }^{4}$ SM Thygerson, ${ }^{5}$ WS Carter, ${ }^{4}$ JD Johnston. ${ }^{1}$ Department of Community Medicine, Kathmandu Medical College, Kathmandu, Nepal; ${ }^{2}$ Department of Community Medicine, Kathmandu University School of Medical Sciences, Kavre, Nepal; ${ }^{3}$ Department of Environment Science and Engineering, School of Science, Kathmandu University, Kavre, Nepal; ${ }^{4}$ Environmental and Occupational Health, Department of Health Science, Brigham Young University, UT, USA; ${ }^{5}$ Environmental Management, University of Findlay, Findlay, OH, USA

\subsection{6/oemed-2018-ICOHabstracts.1244}

Introduction Bricks have been manufactured in Nepal for hundreds of years. Large quantities of hazardous materials including high concentrations of particulate matter are emitted daily from brick kilns. Exposure to these hazardous materials can lead to adverse environmental and human health consequences. This study was conducted to estimate the prevalence of respiratory symptoms/illnesses and the magnitude of respirable and total dust exposures among Nepalese brick kiln workers. Methods Respiratory symptoms/illnesses were evaluated by questionnaire among brickfield workers $(n=400)$ and a referent group of grocery workers $(n=400)$ in Kathmandu valley. Work zones (WZs): green brick moulding (GBM), green brick stacking/carrying (GBS/C), red brick loading/carrying (RBL/C), coal preparation (CP) and firemen (FM) were the similar exposure groups (SEGs) from where personal air samples and interviews were taken, among brickfield workers, personal monitoring was conducted across SEGs for total $(n=89)$ and respirable $(n=72)$. Applying multi-stage probability proportionate to size sampling technique, 16 kilns and 400 brick workers for interview were selected.

Result Chronic cough (14.3\%), phlegm (16.6\%) and bronchitis $(19.0 \%)$ were higher $(\mathrm{p}<0.05)$ among brickfield compared with grocery workers (6.8, 5.8 and 10.8\%). Mean respirable $(5.888 \mathrm{mg} / \mathrm{m} 3)$ and total $(20.657 \mathrm{mg} / \mathrm{m} 3)$ dust exposures were highest for red brick loading tasks. The prevalence of chronic cough, chronic phlegm, chronic bronchitis, wheezing and asthma were significantly higher for other WZs workers $(\mathrm{p}<0.05)$ compared with CP; for GBM: 22.9, 34.6, $15.0 \%$ and 7.5\%; for GBS/C: $13.5,15.8,10.0,8.8$ and $7.5 \%$; for RBL/C: 11.1, 17.1, 27.4, 19.0\% and 11.9\%; for FM: 18.4, $12.5,28.4,4.9$ and $0.0 \%$; and for CP:4.9, 6.3, 13.3, 9.3 and $4.0 \%$ respectively.

Discussion High dust exposures identified in this study may explain the increased prevalence of respiratory symptoms/illnesses among Nepalese brickfield workers, warranting action to reduce exposures. 